\title{
Dieta del pez espada Xiphias gladius Linnaeus, 1758, en aguas oceánicas de Chile central en invierno de 2003
}

\author{
Christian M. Ibáñez ${ }^{1}$, Carlos González ${ }^{2} \&$ Luis Cubillos ${ }^{3}$ \\ ${ }^{1}$ Departamento de Ecología Costera, Facultad de Ciencias, Universidad Católica de la Santísima \\ Concepción. Casilla 297, Concepción, Chile \\ ${ }^{2}$ Departamento de Pesquería, Instituto de Investigación Pesquera. Casilla 350, Talcahuano, Chile \\ ${ }^{3}$ Departamento de Oceanografía, Facultad de Ciencias Naturales y Oceanográficas \\ Universidad de Concepción. Casilla 160-C, Concepción, Chile
}

\begin{abstract}
RESUMEN. Se analiza la dieta del pez espada en tres zonas de pesca en aguas oceánicas de Chile central durante el invierno de 2003, considerando como hipótesis que el tamaño de las presas se incrementa significativamente con el tamaño del depredador, conjuntamente con una disminución del número de presas. El estudio se realizó sobre la base de 48 estómagos de Xiphias gladius con contenido estomacal. Para su análisis se utilizó los descriptores cuantitativos de frecuencia de ocurrencia y método numérico. Se comparó la dieta entre las zonas de muestreo mediante la frecuencia de ocurrencia de las presas. Conjuntamente, se comparó la proporción de peces y cefalópodos, el número y el tamaño de los cefalópodos presas con el tamaño corporal del pez espada. En este estudio se encontró que $X$. gladius es un depredador principalmente teutófago y secundariamente piscívoro, siendo Dosidicus gigas la principal presa en dos de las tres zonas ( $\mathrm{F}=100$ y 75\%), y Onychoteuthis banksii en una de ellas ( $\mathrm{F}=71 \%)$. No se encontraron diferencias significativas en las relaciones de número de presas con el tamaño corporal de X. gladius, y una baja correlación con el tamaño de las presas. La dieta de $X$. gladius varía solamente respecto de la zona de captura de los ejemplares, no siendo relevante el tamaño del depredador. El hábitat del depredador es uno de los factores más importantes a considerar en el estudio de su dieta, ya que sus variaciones afectan la dinámica poblacional de presas y depredadores.
\end{abstract}

Palabras clave: Xiphias gladius, dieta, cefalópodos, tamaño corporal, Pacífico suroriental, Chile.

\section{Diet of the swordfish Xiphias gladius Linnaeus, 1758 in oceanic waters off central Chile in winter 2003}

\begin{abstract}
The diet of swordfish is analyzed in three oceanic fishing zones off central Chile during winter 2003, testing the hypothesis that the body size of prey individuals significantly increases with the size of the predator, and that the number of prey individuals decreases with increase in their size. The study was based on 48 stomachs of Xiphias gladius found to contain identifiable prey remains. The analysis was made using quantitative descriptors of frequency and occurrence of prey items. Diets in the different sampling zones were ranked by frequency and occurrence of prey species. Also determined were the proportions of fishes and cephalopods in the diet, and number and sizes of cephalopods related to body lengths of the swordfish. Results of the study showed that the swordfish preyed primarily on squid, and secondarily on fishes. Dosidicus gigas was the main prey within two of the sampling zones ( $\mathrm{F}=100$ and $75 \%)$, and Onychoteuthis banksii in one zone ( $\mathrm{F}=71 \%)$. The number of prey individuals was not significantly related with the body length of $X$. gladius, but low correlation was noted between swordfish body size and prey size. The diet of $X$. gladius varied only according to the fishing zone, and was not related to the size of the predator. The habitat of the predator is one of the most important factors to be considered in the study of its' diet, since variation in the habitat may affect the population dynamics of both prey and predator.
\end{abstract}

Key words: Xiphias gladius, diet, cephalopods, body size, SE Pacific, Chile. 


\section{INTRODUCCIÓN}

El pez espada (Xiphias gladius Linnaeus, 1758) es un teleósteo pelágico con una distribución geográfica cosmopolita entre los $45^{\circ} \mathrm{N} \mathrm{y} 45^{\circ} \mathrm{S}$ (Palko et al., 1981; Nakamura, 1985; Hernández-García, 1995). En Chile, la pesquería se realiza tradicionalmente por pescadores artesanales, y sólo en la década de los años 90 por una pesquería industrial. La distribución espacial de esta especie está asociada a temperaturas superficiales de $14^{\circ}$ a $18^{\circ} \mathrm{C}$, en aguas de origen oceánico más cálidas que las aguas costeras (Yáñez et al., 1996; Barbieri et al., 1998), encontrándose preferentemente en aguas temperadas mayores a $13^{\circ} \mathrm{C}$ (Nakamura, 1985).

Xiphias gladius es considerado un depredador oportunista que se alimenta de especies pelágicas de crustáceos, cefalópodos y peces (Palko et al., 1981; Nakamura, 1985; Bello, 1991). Su dieta varía ontogenética y estacionalmente (Daza, 2002), como en la mayoría de las especies de peces migratorias que cambian de hábitat y de dieta durante su vida (Smale, 1996). Por esto, se espera que en la dieta de $X$. gladius la proporción de peces y cefalópodos pudiera variar con relación al tamaño corporal, reflejando algún tipo de variación ontogénica en la selectividad de sus presas. De acuerdo con Wootton (1990), dentro de los factores que determinan la selección de las presas por parte del depredador, son importantes las características visuales como el tamaño, contraste con el medio y movimiento. En la dieta de $X$. gladius podría ser importante el tamaño y número de presas consumidas en relación con el tamaño del depredador, debido a que la probabilidad de captura se incrementa con el tamaño de la presa. A su vez, el depredador incrementa el tiempo y costo de captura de la presa (Wootton, 1990). Así, la energía obtenida por unidad de tiempo es maximizada cuando el costo de la captura es minimizado (Wootton, 1990). Además, tanto el tamaño del depredador como de la presa es un atributo que ha sido vinculado directamente con el forrajeo y es importante en la determinación del resultado de las interacciones entre especies (Scharf et al., 2000). En este estudio se considera como hipótesis que el tamaño de las presas se incrementa significativamente con el tamaño del depredador y conjuntamente una disminución del número de presas, debido a una restricción morfológica asociada al tamaño de la boca y volumen estomacal. Si esto se cumple, entonces el número de presas se relacionaría inversamente con el tamaño de las mismas.
Algunos estudios reportan variaciones geográficas en la alimentación del pez espada (Bello, 1991; Hernández-García, 1995; Markaida \& SosaNishizaki, 1998; Daza, 2002), pero dichas variaciones no han sido adecuadamente comparadas. Debido al carácter oportunista de $X$. gladius y sus hábitos de captura de presas, su dieta podría diferir, en términos de la frecuencia de ocurrencia de presas, en cada zona de pesca en una corta escala temporal.

El objetivo de este trabajo es describir la dieta de $X$. gladius en aguas oceánicas de Chile central en invierno de 2003, y analizar las relaciones corporales del depredador y presas.

\section{MATERIALES Y MÉTODOS}

Se recolectaron 48 estómagos de pez espada a bordo de embarcaciones artesanales en las cercanías del archipiélago de Juan Fernández durante el invierno de 2003. Las muestras de estómagos se asignaron a tres zonas (Fig. 1). En la zona 1 se obtuvieron 15 estómagos del 27 de junio al 9 de julio; en la zona 2 se recolectaron 21 estómagos entre el 20 y 29 de julio; y en la zona 3 se recolectaron 12 estómagos los días 22 y 23 de agosto. A bordo de las embarcaciones, a cada ejemplar se le midió la longitud horquilla (desde la mandíbula inferior hasta la horquilla LHMI) en centímetros. Los estómagos fueron fijados en formalina diluida al $10 \%$ para su posterior análisis en laboratorio, donde se procedió a disectar los estómagos para determinar los ítemes presas del contenido estomacal.

Los teleósteos fueron identificados por observación visual, según el grado de digestión. Los restos de huesos se compararon con material de referencia y claves de literatura (Leible \& Miranda, 1989; Falabella et al., 1995). Los cefalópodos fueron identificados mediante material de referencia y claves especializadas (Wolf, 1982; Nesis, 1987). Cuando algunas presas presentaron un alto grado de digestión se cuantificó como restos de peces y/o cefalópodos, según el caso.

Se utilizaron los descriptores cuantitativos descritos por Hyslop (1980), consistentes en el método numérico $(\% \mathrm{~N})$, que representa al número de individuos de un ítem presa determinado como porcentaje del total de individuos de todos los ítemes presa presentes en la muestra, y el método de frecuencia $(\% \mathrm{~F})$, que representa el número de veces que un ítem presa aparece en el total de estómagos con contenido, expresado en porcentaje. Se utilizaron estos 


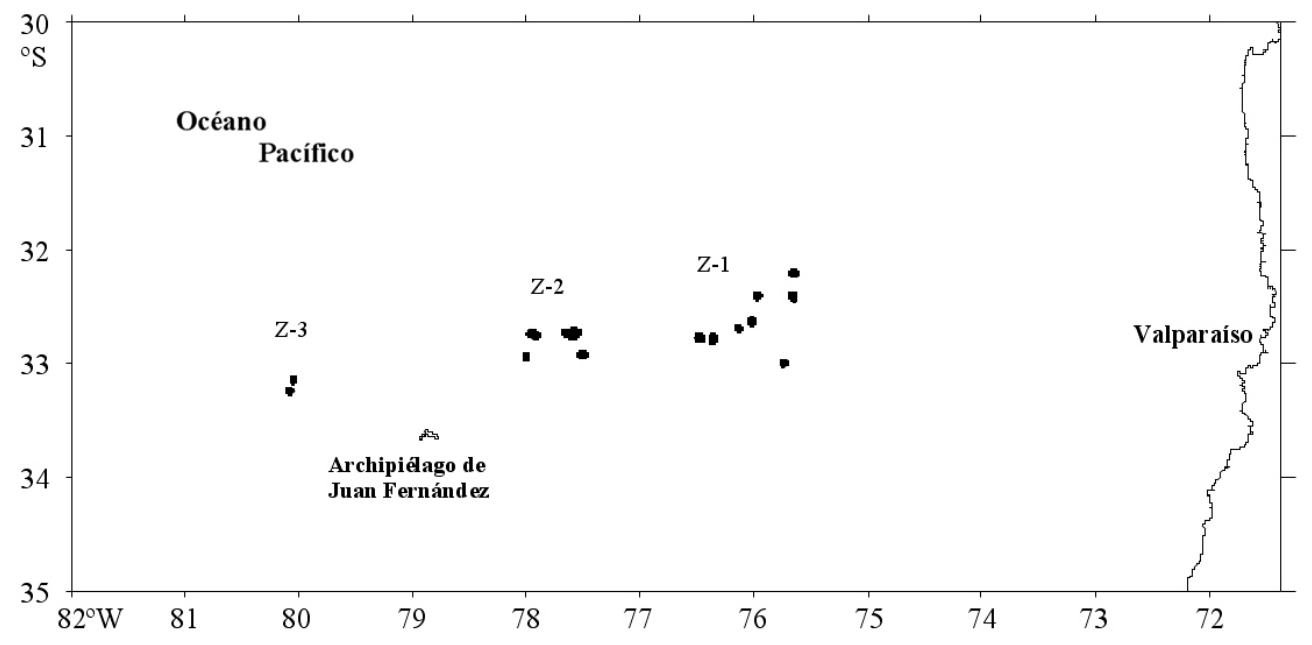

Figura 1. Mapa de las zonas de captura de Xiphias gladius, durante el invierno de 2003.

Figure 1. Fishing zones in which Xiphias gladius was caught in winter 2003.

métodos porque no dependen del grado de digestión de las presas como el peso de las presas y el índice de importancia relativa que son más imprecisos. Los picos o mandíbulas de cefalópodos, encontrados en los estómagos de X. gladius, fueron medidos en su longitud rostral de la mandíbula inferior y/o superior con una precisión de $1 \mathrm{~mm}$. Estas medidas se utilizaron para retrocalcular la longitud del manto (LM, cm) de los ejemplares, con las ecuaciones establecidas por Wolff (1982). No se estimaron los pesos de los cefalópodos ya que no se conocía el peso de los ejemplares de $X$. gladius, para establecer alguna relación. Posteriormente, se realizaron histogramas de frecuencia de tamaños de las principales especies de cefalópodos encontrados en los estómagos.

Se analizó la frecuencia relativa porcentual de las presas agrupadas en peces y cefalópodos por rangos de tamaño corporal de $X$. gladius cada $10 \mathrm{~cm}$ de LHMI. Con el objeto de evaluar diferencias de la dieta con respecto al tamaño corporal del depredador se realizó una tabla de contingencia de máxima verosimilitud (Test G) (Zar, 1984). Conjuntamente, se comparó el número de presas agrupadas en peces y cefalópodos con el tamaño del depredador mediante una correlación de Spearman (Zar, 1984). Para verificar si el depredador poseía alguna preferencia por un determinado tamaño de presas y si esta preferencia se incrementaba con el tamaño corporal del depredador, se realizaron correlaciones de Spearman entre la longitud corporal de las presas y del depre- dador (Zar, 1984), considerando sólo a los cefalópodos, ya que la mayoría de los peces presentaban un alto grado de digestión (solo dos ejemplares estaban completos). Todas las relaciones del tamaño corporal de $X$. gladius con la proporción de peces y cefalópodos, número de presas y tamaño de los cefalópodos en los contenidos estomacales se realizaron con el total de la muestras $(n=48)$, para tener un amplio rango de tamaños de $X$. gladius. Finalmente, para probar la hipótesis propuesta, se relacionó mediante una correlación de Spearman (Zar, 1984), el número de cefalópodos presas con el promedio de su tamaño.

\section{RESULTADOS}

El tamaño promedio de los ejemplares examinados fue de 180,9 cm (rango: 125-250 cm, desviación estándar (DE): 28,8 cm) con una mayor frecuencia alrededor de $180 \mathrm{~cm}$ de LHMI (Fig. 2).

La dieta del pez espada, en la época y zona de estudio, estuvo constituida principalmente por cefalópodos y peces. En la Tabla 1 se detalla el espectro trófico para cada zona de estudio. En la zona 1, la presa más importante en número y frecuencia fue Dosidicus gigas (93,3 y 100\%, respectivamente). En la zona 2, Onychoteuthis banksii y D. gigas fueron las presas más importantes en número (52,6 y $28,6 \%$, respectivamente), y las presas más frecuentes pero en orden inverso $(71,4$ y $66,7 \%)$, seguidas 
por Thyrsites atun $(38,1 \%)$, Stenoteuthis oualaniensis $(28,6 \%)$ y Gonatus antarcticus (19\%). En la zona 3, las presas con mayor porcentaje numérico fueron $O$. banksii (33,8\%), Trachurus murphyi $(23,9 \%)$ y $D$. gigas $(19,7 \%)$, mientras que en frecuencia fueron D. gigas $(75 \%), T$. murphyi $(66,7 \%), O$. banksii $(58,3 \%), T$. atun $(41,7 \%)$, y $S$. oualaniensis (25\%). La frecuencia de ocurrencia de las presas resultó ser dependiente de la zona en que se capturaron los peces espada $\left(\chi^{2}=317,13 ;\right.$ g.1. $\left.=26 ; \mathrm{p}<0,001\right)$.

La proporción de peces y cefalópodos no depende del tamaño del predador $\left(\chi^{2}=3,557\right.$, g.l. $=11, \mathrm{p}=0,98)$ (Fig. 3). Por otro lado, el número de presas de peces y cefalópodos no se correlacionó con el tamaño del depredador $\left(r_{\mathrm{s}}=-0,14, \mathrm{n}=46, \mathrm{p}=0,35 \mathrm{y} \mathrm{r}_{\mathrm{s}}=-0,13, \mathrm{n}=46\right.$, $\mathrm{p}=0,37$, respectivamente) (Fig. 4). La longitud del manto de las principales especies de cefalópodos en los contenidos estomacales de $X$. gladius no se incrementa con el tamaño del depredador en D. gigas $\left(\mathrm{r}_{\mathrm{s}}=0,03, \mathrm{n}=162, \mathrm{p}=\right.$ $0,67)$, S. oualaniensis $\left(\mathrm{r}_{\mathrm{s}}=0,006, \mathrm{n}=11, \mathrm{p}=\right.$ $0,98)$ y $O$. banksii $\left(\mathrm{r}_{\mathrm{s}}=-0,03, \mathrm{n}=118, \mathrm{p}=\right.$ 0,69) (Fig. 5). Sin embargo, al considerar a las presas como un conjunto de cefalópodos se encontró una correlación significativa $\left(\mathrm{r}_{\mathrm{s}}=\right.$ 0,26, $\mathrm{n}=291, \mathrm{p}<0,001)$. Finalmente, el tamaño y número de cefalópodos presas en los estómagos de $X$. gladius no fueron significativamente inversos $\left(r_{s}=-0,14, n=41\right.$, $\mathrm{p}=0,37)$.

La frecuencia de tamaño de las presas $D$. gigas y $O$. banksii se presenta en las figuras 6 y 7, con un promedio de 46,1 cm LM (rango: 18,7-101,1 cm, DE: $10,26 \mathrm{~cm}$ ) y 44,0 cm LM (rango: 18,7-68,8 cm, DE: 7,23 cm), respectivamente. El tamaño de estas presas relativo al tamaño del depredador en porcentaje, alcanzó un promedio de 24,5\% (rango: 10,7-48,3\%, DE: $6,3 \%$ ) para $D$. gigas y $14,3 \%$ (rango: $5,4-$ 24,5\%, DE: 3,5\%) para O. banksii.

\section{DISCUSIÓN}

La dieta de $X$. gladius, en la zona y época de estudio, se compone principalmente de cefalópodos y secundariamente de peces, similar a lo reportado para esta especie en el mar Mediterráneo (Bello, 1991), golfo de

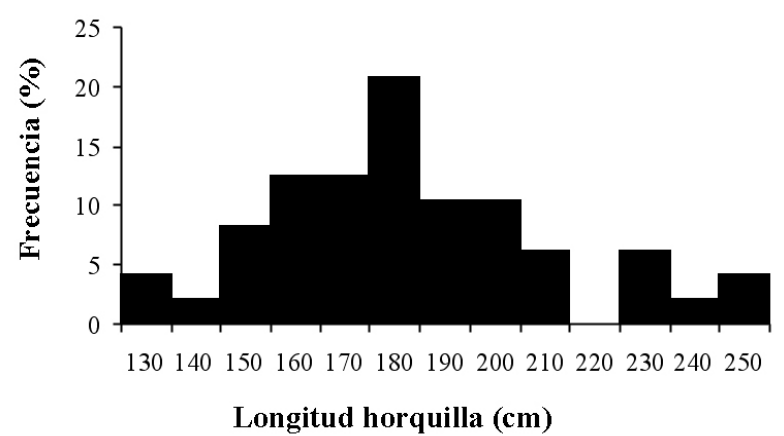

Figura 2. Distribución de frecuencia de tamaños de Xiphias gladius.

Figure 2. Length-frequency distribution of Xiphias gladius.

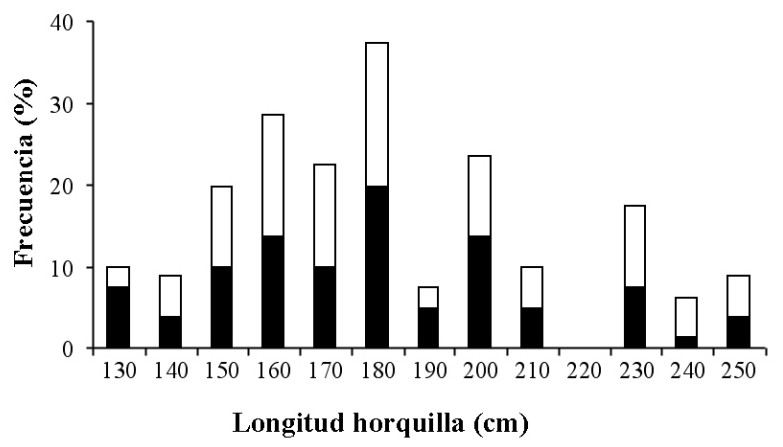

Figura 3. Frecuencia de ocurrencia $(\%)$ de cefalópodos (negro) y peces (blanco) en los contenidos estomacales de Xiphias gladius por clases de tamaños.

Figure 3. Frequency of occurrence (\%) of cephalopods (black) and teleosts (white) in the stomach contents of Xiphias gladius by length classes.

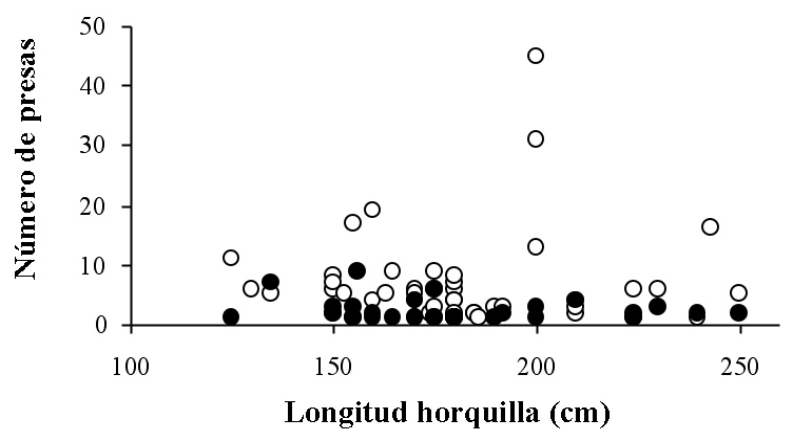

Figura 4. Número de presas de cefalópodos (círculos blancos) y peces (círculos negros) en los contenidos estomacales de Xiphias gladius por clases de tamaños.

Figure 4. Preys number of cephalopods (open circles) and teleosts (closed circles) in the stomach contents of Xiphias gladius by length classes. 


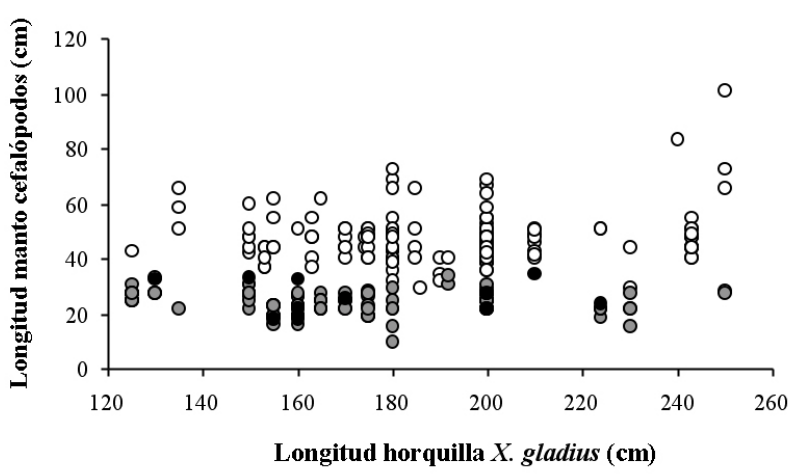

Figura 5. Relación entre la longitud del manto (LM) de los cefalópodos (Dosidicus gigas, $\mathbf{n}=162$ círculos blancos; Onychoteuthis banksii, $\mathbf{n}=118$ círculos grises; Stenoteuthis oualaniensis, $\mathbf{n}=11$ círculos negros) y la longitud horquilla mandíbula inferior (LHMI) de Xiphias gladius.

Figure 5. Relationships between of the mantle length (LM) of cephalopods (Dosidicus gigas, $\mathbf{n}=162$ open circles; Onychoteuthis banksii, $\mathbf{n}=118$ grey circles; Stenoteuthis oualaniensis, $\mathbf{n}=11$ closed circles) and fork jaw lower length (LHMI) of Xiphias gladius.

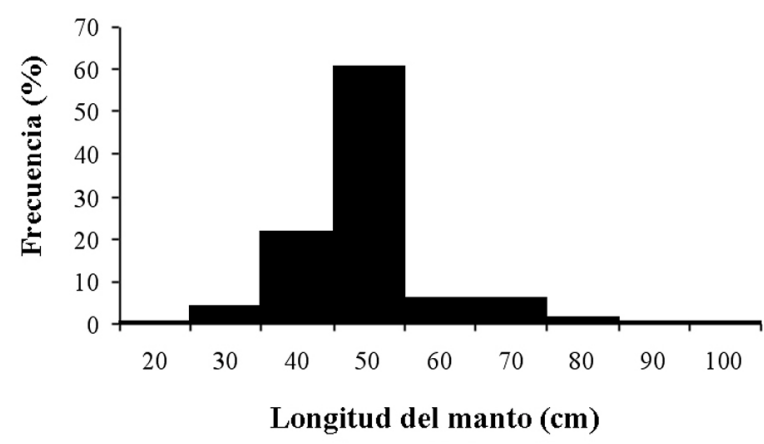

Figura 6. Distribución de frecuencia de tamaños de Dosidicus gigas encontrados en los contenidos estomacales de Xiphias gladius.

Figure 6. Length-frequency distribution of Dosidicus gigas in the stomach contents of Xiphias gladius.

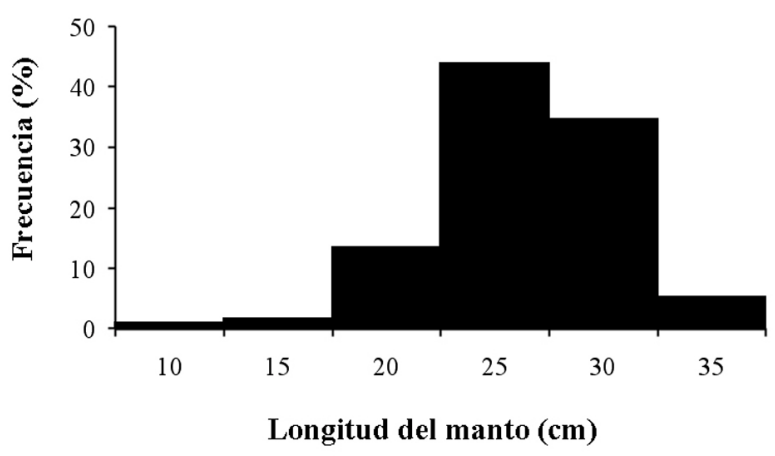

Figura 7. Distribución de frecuencia de tamaños de Onychoteuthis banksii encontrados en los contenidos estomacales de Xiphias gladius.

Figure 7. Length-frequency distribution of Onychoteuthis banksii in the stomach contents of Xiphias gladius.
California (Markaida \& Sosa-Nishizaki, 1998), Atlántico norte (Guerra et al., 1993), costas de Portugal (Moreira, 1990) e islas Canarias (Hernández-García, 1995). Lo anterior es coherente con la importancia que tienen los cefalópodos en los ecosistemas marinos (Amaratunga, 1983) y en la dieta de los peces (Smale, 1996).

Al parecer, de acuerdo con los resultados obtenidos, $X$. gladius no disminuye el número de presas, con relación al tamaño corporal, a pesar que éstas incrementan su tamaño, ya que se observó concordancia entre el tamaño de las presas y su tamaño corporal cuando las presas de cefalópodos fueron tratadas en conjunto. Sin embargo, para el rango de tamaños analizados, la proporción de peces y cefalópodos en la dieta no varía durante su ontogenia. Podría existir, teóricamente, un tamaño óptimo de las presas disponibles en el ambiente, en el cual se minimiza el costo y tiempo de captura, pero que seguramente presentarían una baja probabilidad de captura y energía obtenida, no ajustándose a lo señalado por la teoría de forrajeo óptimo (Hughes, 1980). Contrariamente a la escasa variación ontogénica en la dieta, la zona de pesca es probablemente el factor más importante en las variaciones de la alimentación de esta especie, lo que podría estar relacionado con la abundancia espacial y temporal de sus presas. Algo similar se ha encontrado en la dieta del atún Thunnus maccoyi en Tasmania, donde su dieta no cambia durante su ontogenia 
Tabla 1. Composición dietaria de Xiphias gladius en las zonas de estudio. N: número de presas, F: frecuencia de presas, n: número de estómagos analizados.

Table 1. Food composition of Xiphias gladius in the study areas. N: number of preys, F: frequency of preys, $n$ : number od stomachs analyzed.

\begin{tabular}{|c|c|c|c|c|c|c|}
\hline & \multicolumn{2}{|c|}{$\begin{array}{c}\text { Zona 1 } \\
32^{\circ} 30^{\prime} S, 7^{\circ} 00^{\prime} W \\
(n=15)\end{array}$} & \multicolumn{2}{|c|}{$\begin{array}{c}\text { Zona } 2 \\
3^{\circ} 45 ' S, 7^{\circ} 50 \text { 'W } \\
(n=21)\end{array}$} & \multicolumn{2}{|c|}{$\begin{array}{c}\text { Zona } 3 \\
\left.\text { 33'10'S, 80'10'W }^{\circ}{ }^{\circ}=12\right)\end{array}$} \\
\hline & $\% \mathbf{N}$ & $\% \mathbf{F}$ & $\% \mathbf{N}$ & $\% \mathbf{F}$ & $\% \mathbf{N}$ & $\% \mathbf{F}$ \\
\hline \multicolumn{7}{|l|}{ CEPHALOPODA } \\
\hline Dosidicus gigas & 93,3 & 100,0 & 28,5 & 66,7 & 19,8 & 75,0 \\
\hline Stenoteuthis oualaniensis & 1,0 & 6,7 & 4,0 & 28,6 & 4,2 & 25,0 \\
\hline Todarodes filippovae & 1,0 & 6,7 & & & & \\
\hline Onychoteuthis banksii & 1,9 & 6,7 & 52,6 & 71,4 & 33,8 & 58,3 \\
\hline Gonatus antarcticus & & & 2,9 & 19,0 & & \\
\hline Loligo gahi & & & 2,9 & 4,8 & & \\
\hline \multicolumn{7}{|l|}{ TELEOSTEI } \\
\hline Trachurus murphyi & 1,0 & 6,7 & & & 23,9 & 66,7 \\
\hline Thyrsites atun & & & 5,7 & 38,1 & 8,5 & 41,7 \\
\hline Scomber japonicus & & & 1,1 & 4,8 & & \\
\hline Seriola peruana & 1,0 & 6,7 & & & & \\
\hline Argyropelecus sp. & & & & & 7,0 & 8,3 \\
\hline Teleostei 1 & 1,0 & 6,7 & & & 1,4 & 8,3 \\
\hline Teleostei 2 & & & 1,7 & 4,8 & & \\
\hline Teleostei 3 & & & 0,6 & 4,8 & 1,4 & 8,3 \\
\hline
\end{tabular}

en composición, diversidad, peso, tamaño y número de presas, pero sí geográficamente (Young et al., 1997). Sin embargo, Scott \& Tibbo (1968) encontraron una relación positiva entre el volumen del contenido estomacal y el tamaño corporal de $X$. gladius. En la Zona 1, geográficamente, dominó la presencia de D. gigas, en relación a las otras zonas, esto tal vez debido a que en las zonas donde la jibia es más abundante, otras especies de calamares y peces escapan de su presencia, ya que es un depredador voraz que consume principalmente peces y calamares (Nigmatullin et al., 2001). Estudios futuros deben cuantificar la oferta ambiental de posibles presas del pez espada en las zonas de captura, para comparar con lo encontrado en el contenido gástrico, y así aclarar si $X$. gladius es una especie oportunista o selectiva.

El número de presas de D. gigas y $S$. oualaniensis casi siempre fue bajo, debido al gran tamaño de éstas, a excepción de $O$. banksii que fue comparativa- mente la presa más numerosa y más pequeña. Una posible explicación que se plantea para elucidar el tamaño de los cefalópodos consumidos por $X$. gladius, es que ataca cardúmenes de calamares con una distribución de tamaños muy acotada. Lo mismo ocurre con los peces, ya que consume especies que viven en agregaciones como Trachurus murphyi y Thyrsites atun. Ya que la mayoría de los peces y cefalópodos encontrados en los estómagos estaban destruidos y en algunos casos cortados en dos, debido al uso de su apéndice rostral para matar a sus presas según lo reportado anteriormente por Scott $\&$ Tibbo (1968), se supone que atacan a gran velocidad los cardúmenes de presas, lo que les permitiría alimentarse de presas tan grandes como $D$. gigas, que alcanza cerca del $50 \%$ del tamaño corporal de X. gladius.

La dieta de un pez refleja el alimento disponible en el ambiente, razón por la cual la dieta del pez espada puede ser utilizada para conocer la abundan- 
cia relativa de las presas a través de su contenido estomacal (Wootton, 1990; Smale, 1996). Así, el pez espada puede ser un estimador de la presencia, abundancia y diversidad de peces, cefalópodos y otros organismos que podría consumir en diferentes escalas espacio-temporales. Importante es relacionar esto con las variaciones ambientales, ya que afectan la dinámica poblacional de las presas y en consecuencia producen cambios en la dieta del depredador, porque las presas disponibles pueden migrar, reducir y/o aumentar su densidad en función de la variación ambiental. Esto se refleja en la dieta de $X$. gladius, que en años previos estaba constituida mayoritariamente de peces (Barbieri et al., 1998; Daza, 2002), y en el año 2003 se compuso principalmente de calamares, sobre todo de D. gigas, que es una especie que incrementó notablemente su presencia y abundancia relativa en la zona centro-sur de Chile en los últimos 3 años (Cubillos et al., 2004).

\section{AGRADECIMIENTOS}

Los autores agradecen a la tripulación de las embarcaciones Moroni y Nefi, por facilitar la recolección de las muestras de estómagos; reconocen la ayuda de R. Mena del Instituto de Investigación Pesquera, INPESCA, en la determinación específica de los peces en el contenido estomacal del pez espada.

\section{REFERENCIAS}

Amaratunga, T. 1983. The role of cephalopods in the marine ecosystem. En: J.F. Caddy (ed.). Advances in assessment of world cephalopods resources. FAO Fish. Tech. Pap., 231: 379-415.

Barbieri, M.A., C. Canales, V. Correa, M. Donoso, A. González, B. Leiva, A. Montiel \& E. Yáñez. 1998. Development and present state of the swordfish, Xiphias gladius, fishery in Chile. En: I. Barrett, O. Sosa-Nishizaki \& N. Bartoo (eds.). Biology and fisheries of swordfish, Xiphias gladius. NOAA Tech. Rep. NMFS, 142: 1-10.

Bello, G. 1991. Role of cephalopods in the diet of the swordfish, Xiphias gladius, from the eastern Mediterranean sea. Bull. Mar. Sci., 49(1-2): 312-324.

Cubillos, L., C. Ibáñez, C. González \& A. Sepúlveda. 2004. Pesca de jibia (Dosidicus gigas) con red de cerco entre la V y X Regiones, año 2003. Informe Final Pesca de Investigación. Instituto de Investigación Pesquera, Talcahuano, Chile, 52 pp.

Daza, E. 2002. Dinámica trófica del pez espada Xiphias gladius Linnaeus, 1758, asociada a la pesquería del recurso en el Pacífico Sur Oriental. Tesis de Biología Marina, Universidad de Concepción, 120 pp.

Falabella, F., R. Meléndez \& M.L. Vargas. 1995. Claves osteológicas para peces de Chile central, un enfoque arqueológico. Editorial Artegrama, Santiago, 208 pp.

Guerra, A., F. Simon \& A. González. 1993. Cephalopods in the diet of the swordfish, Xiphias gladius, from the northeastern Atlantic Ocean. En: T. Okutani, R.K. O’Dor \& T. Kubodera (eds.). Recent advances in cephalopod fisheries biology. Tokai University Press, Tokyo, pp. 159-164.

Hernández-García, V. 1995. The diet of the swordfish Xiphias gladius Linnaeus, 1758, in the central east Atlantic, with emphasis on the role of cephalopods. US Fish. Bull., 93: 403-411.

Hughes, R.N. 1980. Optimal foraging theory in the marine context. Oceanogr. Mar. Biol., 18: 423-481.

Hyslop, J. 1980. Stomach contents analysis: a review of methods and their application. J. Fish Biol., 17: 411-429.

Leible, M. \& P. Miranda. 1989. El otolito sagitta en el reconocimiento de diferentes especies de teleósteos de la costa central de Chile. Bol. Soc. Biol., Concepción, 60: 149-160.

Markaida, U. \& O. Sosa-Nishizaki. 1998. Food and feeding habits of swordfish, Xiphias gladius L., off western Baja California. En: I. Barrett, O. SosaNishizaki \& N. Bartoo (eds.). Biology and fisheries of swordfish, Xiphias gladius. NOAA Tech. Rep. NMFS, 142: 245-259.

Moreira, F. 1990. Food of the swordfish, Xiphias gladius Linnaeus, 1758, off the Portuguese coast. J. Fish Biol., 36: 623-624.

Nakamura, I. 1985. Billfishes of the world. An annotated and illustrated catalogue of marlins, sailfishes, spearfishes and swordfishes known to date. FAO Fish. Synop., 125(5): 1-65.

Nesis, K.N. 1987. Cephalopods of the world. T.F.H. Publications, New Jersey, 351 pp. 
Nigmatullin, Ch.M., K.N. Nesis \& A.I. Arkhipkin. 2001. A review of the biology of the jumbo squid Dosidicus gigas (Cephalopoda: Ommastrephidae). Fish. Res., 54: 9-19.

Palko, B.J., G.L. Beardsley \& W.J. Richards. 1981. Synopsis of the biology of the swordfish, Xiphias gladius Linnaeus. FAO Fish. Synop., 127: 1-21.

Scharf, F.S., F. Juanes \& R.A. Rountree. 2000. Predator size-prey size relationships of marine fish predators: interspecific variation and effects of ontogeny and body size on trophic-niche breadth. Mar. Ecol. Prog. Ser., 208: 229-248.

Scott, W.B. \& S.N. Tibbo. 1968. Food and feeding habits of swordfish, Xiphias gladius, in the western north Atlantic. J. Fish. Res. Bd., Canada, 25(5): 903-919.

Smale, M.J. 1996. Cephalopods as preys. IV. Fishes. Phil. Trans. Roy. Soc. London B., 351: 1067-1081.
Yáñez, E., C. Silva, M.A. Barbieri \& K. Nieto. 1996. Pesquería artesanal de pez espada y temperatura superficial del mar registrada con satélites NOAA en Chile central. Invest. Mar., Valparaíso, 24: 131144.

Young, J.W., T.D. Lamb, D. Le, R.W. Bradford \& A.W. Whitelaw. 1997. Feeding ecology and interannual variations in the diet of southern bluefin tuna, Thunnus maccoyi, in relation to coastal and oceanic waters of eastern Tasmania, Australia. Environ. Biol. Fish., 50: 275-291.

Wolff, G. 1982. A beak key for eight eastern tropical Pacific cephalopod species with relationships between their beak dimensions and size. US Fish. Bull., 26(2): 357-370.

Wootton, R.J. 1990. Ecology of teleost fishes. Chapman and Hall, London, 404 pp.

Zar, J.H. 1984. Biostatistical analysis. Prentice-Hall, New York, 718 pp. 